\author{
Research Fellow Jeonggyu HUH, PhD \\ Computational Sciences, Korea Institute for Advanced Study \\ Seoul, Republic of Korea \\ E-mail: aifina2018@kias.re.kr \\ Assistant Professor Geonwoo KIM, PhD (Corresponding author) \\ School of Liberal Arts \\ Seoul National University of Science and Technology \\ Seoul, Republic of Korea \\ E-mail:geonwoo@seoultech.ac.kr
}

\title{
BARRIER OPTION PRICING WITH HEAVY TAILED DISTRIBUTION
}

\begin{abstract}
Under the Generalized Extreme Value (GEV) model, Markose and Alerton (2011) derived the analytic form solutions for vanilla options, and also removed the distortion of the market only with an additional parameter. In this paper, we use the technique in Rubinstein and Reiner (1991) to get the analytic form solutions for barrier options by introducing the Corrected BS (CBS) modelthe BS model close to the GEV model. By introducing CBS volatility we show that barrier option prices are continuous with respect to barriers under the GEV model. In addition, we present that the proposed model outdoes the BS model.

Keywords: Barrier option pricing, Heavy tailed distribution, Generalized extreme value (GEV) distribution, Global credit crisis.
\end{abstract}

\section{JEL Classification: C58, G01, G13}

\section{Introduction}

According to Jackwerth and Rubinstein (1996), the stock market crash of October 1987 was an extreme event with probability of $10^{-160}$, which is virtually impossible with the Gaussian type thin-tailed distribution models. Two years later, on October 13, 1989, the S\&P 500 index fell about $6 \%$, which has a probability of $2.7 \times 10^{-7}$ and should happen only once in 14,756 years under the same hypothesis. Even though Gaussian type thin-tailed distributions play an essential role in mathematical finance such as Black-Scholes option pricing or the calculation of Value at Risk, it is time to rethink the early suggestion by Mandelbrot (1963) on the possibility of heavy tails in financial data (Kim and Kim, 2014).

The celebrated Black-Scholes (BS) model (1973) has been widely used in derivatives markets, because the model can evaluate plain vanilla options in markets with simple intuitive parameters. However, when the markets fluctuate 
sharply, as the BS model is unable to reflect the skewness and the kurtosis of the markets, the difference between the values of the vanilla call options based on the BS model and the actual prices of the markets grows rapidly. Moreover, when the distribution is skewed to the left, there are serious mismatches in valuating downand-in barrier options based on the BS model assumption since it underestimates the probability of reaching the barrier.

Recently, Markose and Alentorn (2011) proposed a new vanilla option pricing model based on Generalized Extreme Value (GEV) distribution. Extreme value theory has been widely used in hydrology, climatology and insurance areas (Embrechts et al., 1997, Gabaix et al., 2003, Hosking and Wallis, 2005). At the heart of the theory, there are two types of distributions, GEV and Generalized Pareto (GPD) distributions, which can be justified by some limit theorems. ${ }^{1}$

The barrier option pricing formula, on the other hand, was first solved by Merton (1973) as an application of the Black-Scholes-Merton differential equation in Section 9 of his paper. In his paper, he used the so-called method of image for solving the partial differential equation, which requires quite advanced mathematics. Later on, Rubinstein and Reiner (1991) found a much easier way to obtain the same solutions by breaking down the integrations in the risk-neutral valuation.

This paper takes the aforementioned two ideas together, GEV-based option pricing and breaking down the integrations, to derive explicit barrier option pricing formulas based on the GEV distribution. The derived formulas are numerically comparable to the barrier option pricing formulas based on BS assumptions when the markets are stable. However, they over/under-price the BS based barrier option prices when the markets move sharply, indicating that they are capable of capturing the skewness and the kurtosis of the underlying distributions of the market participants.

\section{A Brief Review of GEV distribution}

To introduce the GEV briefly, Figure 1(b) shows the probability density function of GEV distributions with various choices for the shape parameter $\xi$. Note that we can reflect the skewness and the kurtosis ${ }^{2}$ of the return distribution as we control the parameter $\xi$.

The similarity between normal distribution and appropriately chosen GEV distribution $^{3}$ is verified by Figure 2(a). Figure 2(b) shows the probability of hitting barrier according to each barrier when current underlying asset is 142 in the Figure 2(a) distribution.

\footnotetext{
${ }^{1}$ Fisher-Tippett-Gnedenko theorem and Pickands-Balkema-de Haan theorem

${ }^{2}$ skewness of the GEV distribution $=\left(g_{3}-3 g_{1} g_{2}+2 g_{1}^{3}\right) /\left(g_{2}-g_{1}^{2}\right)^{3 / 2}$, kurtosis of the GEV distribution $=\left(g_{4}-4 g_{1} g_{3}+6 g_{2} g_{1}^{2}-3 g_{1}^{4}\right) /\left(g_{2}-g_{1}^{2}\right)^{2}-3$, where $g_{k}=\Gamma(1-k \xi)$

${ }^{3}$ approximation error $=\mid($ pdf of GEV distribution) $-($ pdf of normal distribution $) \mid \div$ (pdf of GEV distribution)
} 
Barrier Option Pricing with Heavy Tailed Distribution

Even though normal distribution is similar to GEV distribution, the probability of normal distribution to breach the upper barrier is underestimated or overestimated to GEV distribution. It means there is a chance that the BS model can undervalue or overvalue OTC derivatives like barrier options, in spite of similar fitting results in exchange-traded derivatives. Furthermore, there is a higher risk to make wrong valuation when the two distributions are not similar.

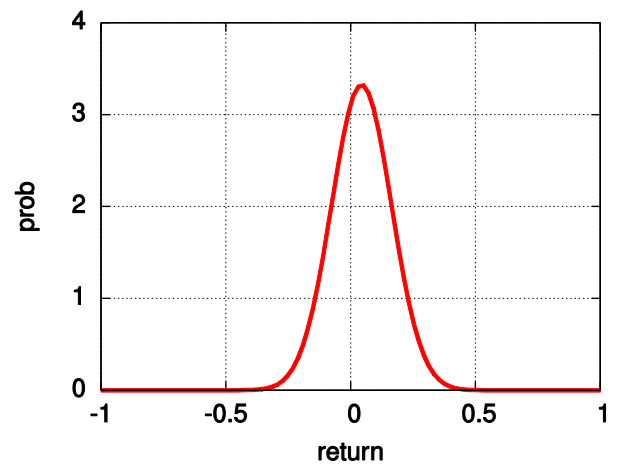

(a) normal distribution

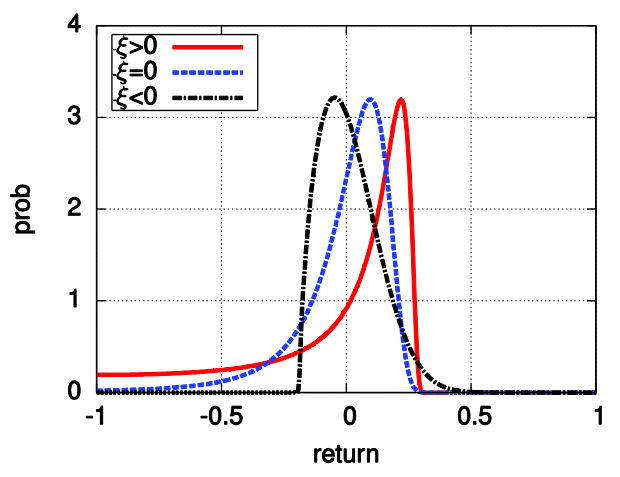

(b) GEV distribution

Figure 1: Pdfs of normal and GEV Distribution

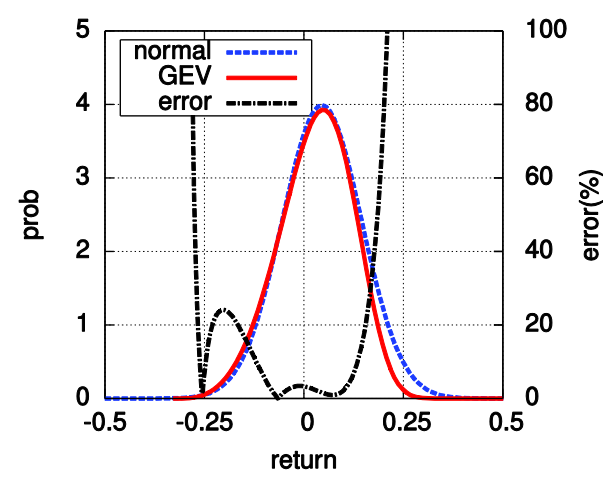

(a) pdfs and error

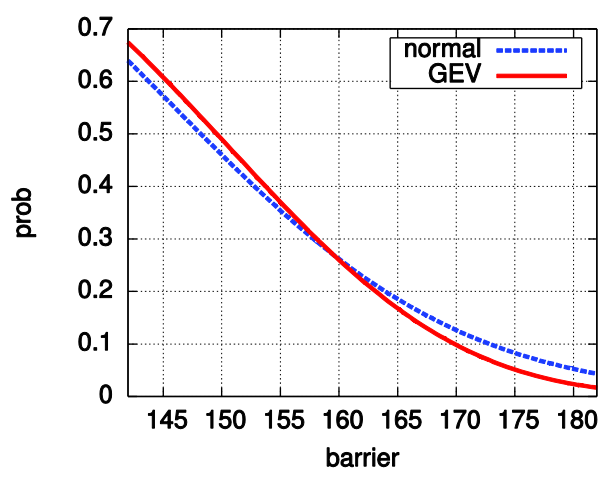

(b) prob to hit upper barriers

Figure 2: Probability to hit barriers when GEV distribution is approximated to normal distribution

\section{Assumptions of the Valuation Model}

To model extreme economic losses, Markose and Alerton (2011) supposed that simple negative returns have the GEV distribution. Under the supposition, it showed that the parameters in the model should satisfy a particular equation in order to rule out arbitrage possibilities. 


\section{Assumption 1}

Let a time interval $[t, T]$ be given: in this paper, all options below are assumed to be priced at $t$ and expire at $T$. We consider an underlying stock with price process $S_{u}(u \in[t, T])$. We assume that simple negative return $L_{t}^{T}=-\left(S_{T}-S_{t}\right) /$ $S_{t}$ has a GEV distribution, that is, $L_{t}^{T} \sim G E V(\mu, \sigma, \xi)$.

In an arbitrage-free economy, where we place ourselves henceforward, the three parameters $\mu, \sigma$, and $\xi$ should satisfy the equation

$$
\mu=1-\frac{F_{t}^{T}}{S_{t}}-\left[\frac{\Gamma(1-\xi)-1}{\xi}\right] \sigma \quad(\xi \neq 0)
$$

Here $F_{t}^{T}$ is the stock futures price at $t$ with maturity $T$ ( Markose and Alerton, 2011). Moreover, a relation among the parameters can be also suggested in the case of $\xi=0$ by $\mu=1-F_{t}^{T} / S_{t}-\tilde{\gamma} \sigma$, where $\tilde{\gamma}$ is the Euler-Mascheroni constant.

The price for a barrier option ${ }^{4}$ is highly sensitive to the distributions of stock returns $s^{5}$ before maturity as well as to the one at maturity, models of which the stochastic process plays a crucial role in establishing. Unlike the BS model, however, it has yet to be confirmed whether there exists a stochastic differential equation which the process satisfies, to the best of the authors' knowledge.

By and large, the implied return distribution at maturity obtained from a model other than the BS model in a market for exchange-traded derivatives does not necessarily have a normal distribution. But a central neighborhood of the implied one still can be approximated by the one derived from the BS model and therein lies our major premise: we presuppose that for a barrier option, a central neighborhood of the return distribution at maturity under the condition that the underlying stock price crosses the barrier before maturity also can be fit by the one under the same condition except that $S_{u}$ would follow a geometric Brownian motion. For the sake of conciseness, it would be timely for us to name the return distributions present in our postulation: from here on, the former is called a GEVbased conditional return distribution and the latter distribution a normal-based conditional return distribution. Further, we let $\tau_{B}$ denote the first instant that the underlying price breaches the barrier at.

The above hypothesis enables us to do without any stochastic process by dint of works of Rubinstein and Reiner (1991) and Shreve (2008), which show that the density function of a normal-based conditional return distribution ${ }^{6}$ is

$$
g(x ; \sigma)=\frac{1}{\sigma \sqrt{2 \pi \tau}} \exp \left\{\frac{2 v \alpha}{\sigma^{2}}\right\} \exp \left\{-\left[\frac{x-2 \alpha-v \tau}{\sigma \sqrt{2 \tau}}\right]^{2}\right\}, \alpha=\ln \frac{B}{S_{t}}, v=r-\frac{1}{2} \sigma^{2}, \tau=T-t
$$

\footnotetext{
${ }^{4}$ All barrier options involved herein are assumed to offer no rebate.

5 Throughout the article, unless otherwise specified, here 'return' always means the log-return.

${ }^{6}$ Technically, $g$ does not satisfy the definition of probability density function. It is indeed positive, but the integral of it on the real line is smaller than 1 . For convenience, $g$ is considered as a density here.
} 


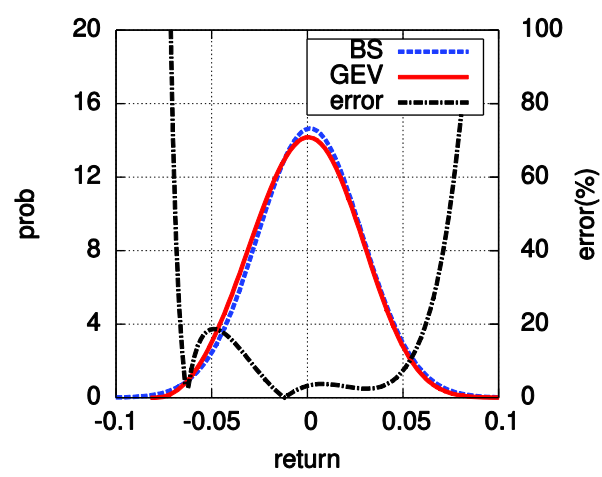

(a) Whole interval

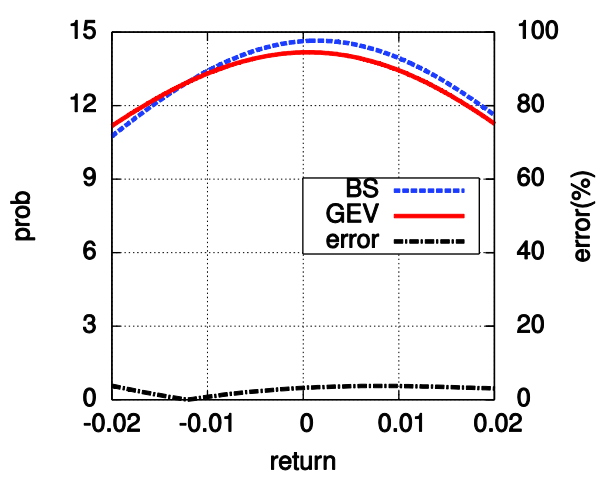

(b) Central neighborhood

Figure 3: PDFs of the GEV model and the BS model when a market is stable ${ }^{7}$

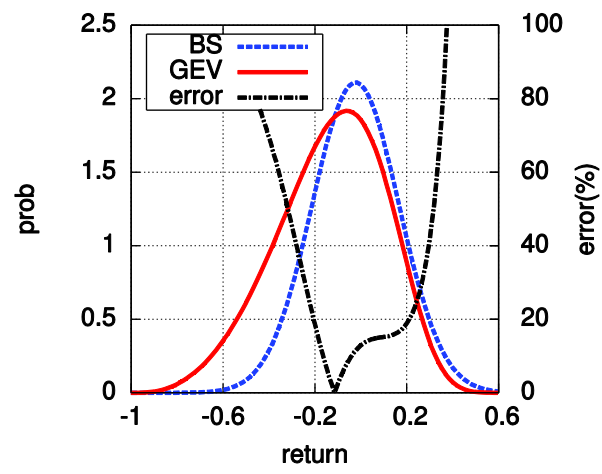

(a) whole interval

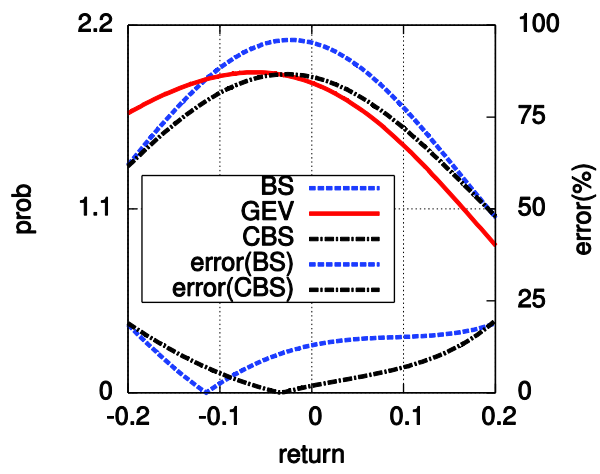

(b) Central neighborhood

Figure 4: PDFs of the GEV model and the BS model when a market is unstable ${ }^{8}$

Figure 3(a) depicts the density function of the stock return from the GEV model along with that from the BS model in a stable market for exchange-traded derivatives, whereas Figure 4(a) does in an unstable one. In the stable market, those functions look alike. On the other hand, however, shown is an obtrusive gap between them in the unstable market.

The same is true of their central neighborhoods which Figures 3(b) and 4(b) illustrate. We can, however, adjust the volatility of the BS model in order that the approximation error in the unstable market be as small as possible. Hereafter we refer to such an adjusted BS volatility as a Corrected BS volatility (CBS volatility) and such an adjusted BS model as a Corrected BS model (CBS model). At first sight, these might be nebulous, but as will be described in Section 5, they will have

${ }^{7}$ Based on the data on KOSPI 200 index call option prices, April 2014

${ }^{8}$ Based on the data on KOSPI 200 index call option prices, October 2008 
Jeonggyu Huh, Geonwoo Kim

been derived from specific formulas and thus will rather turn out to be intrinsic. We sum up and restate the aforementioned discussion as Assumption 2 for future reference.

\section{Assumption 2}

For a barrier option, a central neighborhood of the distribution of the log-return at maturity $r_{t}^{T}=\ln \left(S_{T} / S_{t}\right)$ conditional on having hit the barrier also can be approximated by one under the same condition except that the underlying stock price process would act as the counterpart in the BS model:

$$
\frac{d P\left(r_{t}^{T}<r \mid \tau_{B} \leq T\right)}{d r} \approx g\left(r ; \sigma_{C B S}\right)(|r| \ll 1)
$$

Here $\sigma_{C B S}$ denotes the CBS volatility.

\section{Pricing Formulas of Barrier Options}

The goal of this section is to show how we tried to combine the accuracy of GEV model and the simplicity of the BS model as regards pricing barrier options. To start with, as mentioned earlier, Markose and Alerton (2011) produced the closedform solutions for the GEV-based vanilla option pricing model for $\xi>0$. We omit the proofs of valuation for $\xi<0$ and $\xi=0$ because they are similar to the case for $\xi>0$.

(a) The pricing formula for vanilla call options ${ }^{9}$

$$
\begin{aligned}
& C_{t}(K) \\
& =\left\{\begin{array}{cc}
e^{-r(T-t)}\left[S_{t}\left\{\left(1-\mu+\frac{\sigma}{\xi}\right) \exp \left(-H^{-1 / \xi}\right)-\frac{\sigma}{\xi} \Gamma\left(1-\xi, H^{-1 / \xi}\right)\right\}-K \exp \left(-H^{-1 / \xi}\right)\right] & (\xi \neq 0) \\
e^{-r(T-t)}\left[S_{t}\left\{(1-\mu+\widehat{H} \sigma) \exp \left(-e^{\widehat{H}}\right)+\sigma \Gamma\left(0, e^{\widehat{H}}\right)\right\}-K \exp \left(-e^{\widehat{H}}\right)\right] & (\xi=0)
\end{array}\right.
\end{aligned}
$$

Here $H=1+(\xi / \sigma)\left(1-K / S_{t}-\mu\right), \widehat{H}=-\left(1-K / S_{t}-\mu\right) / \sigma$, and $C_{t}(K)_{G E V}$ is the price of a vanilla call option with strike price $K$ for the GEV model at $t$.

In a similar manner to their calculation, we can draw a closed-form expression of the below integral:

(b) An item in aid of the analytic form valuation of barrier call options under the GEV model

$$
\begin{aligned}
& \tilde{C}_{t}(K, B)_{G E V}=e^{-r(T-t)} \int_{\ln \left(B / S_{t}\right)}^{\infty}\left(S_{t} e^{u}-K\right) f(u) d u \\
& \quad=e^{-r(T-t)}\left[S_{t}\left\{\left(1-\mu+\frac{\sigma}{\xi}\right) \exp \left(-H_{1}{ }^{-1 / \xi}\right)-\frac{\sigma}{\xi} \Gamma\left(1-\xi, H_{1}^{-1 / \xi}\right)\right\}-K \exp \left(-H_{1}^{-1 / \xi}\right)\right](\xi \neq 0)
\end{aligned}
$$

Here $H_{1}=1+(\xi / \sigma)\left(1-B / S_{t}-\mu\right)$ and $f$ isthedensity function of thelogreturn $r_{t}^{T}$ deduced from $L_{t}^{T} \sim G E V(\mu, \sigma, \xi)$ in Assumption 1 . Note that $C_{t}(K)_{G E V}=$ $\tilde{C}_{t}(K, B)_{G E V}$ if $B \leq K$.

${ }^{9} \Gamma(s, x)=\int_{x}^{\infty} t^{s-1} e^{-t} d t$ : (upper) incomplete gamma function 
Assumption 2 allows us to apply techniques in Rubinstein and Reiner (1991) to the valuation problems. To be more specific, we are going to express the situation where the payoff of a barrier option is non-zero as a combination of several simple cases and partition the domain of the integration along the expression. The below formulas are a portion of its results, which will help in constructing the pricing formulas of barrier options later.

(c) Ad-hoc terms which will be used on barrier option pricing

$$
\begin{aligned}
e^{-r(T-t)} \int_{\ln \left(K / S_{t}\right)}^{\infty}\left(S_{t} e^{u}-K\right)^{+} g(u ; \sigma) d u & =C D(y ; \sigma ; \varphi=1, \eta=1) \\
e^{-r(T-t)} \int_{\ln \left(B / S_{t}\right)}^{\infty}\left(S_{t} e^{u}-K\right)^{+} g(u ; \sigma) d u & =C D\left(y_{1} ; \sigma ; \varphi=1, \eta=1\right) \\
e^{-r(T-t)} \int_{-\infty}^{\ln \left(K / S_{t}\right)}\left(S_{t} e^{u}-K\right)^{+} g(u ; \sigma) d u & =C D(y ; \sigma ; \varphi=1, \eta=-1) \\
e^{-r(T-t)} \int_{-\infty}^{\ln \left(B / S_{t}\right)}\left(S_{t} e^{u}-K\right)^{+} g(u ; \sigma) d u & =C D\left(y_{1} ; \sigma ; \varphi=1, \eta=-1\right)
\end{aligned}
$$

Here the function $C D$ and the constantsy, $y_{1}$ are as follows.

$$
\begin{gathered}
C D(x ; \sigma ; \varphi, \eta)=\varphi S_{t}\left(B / S_{t}\right)^{2 \lambda} N(\eta x) \\
-\varphi K e^{-r(T-t)}\left(B / S_{t}\right)^{2 \lambda-2} N(\eta x-\eta \sigma \sqrt{T-t}) \\
y=\frac{\ln \left(B^{2} / S_{t} K\right)}{\sigma \sqrt{T-t}}+\lambda \sigma \sqrt{T-t}, y_{1}=\frac{\ln \left(B / S_{t}\right)}{\sigma \sqrt{T-t}}+\lambda \sigma \sqrt{T-t}, \lambda=\frac{1}{2}+\frac{r}{\sigma^{2}}
\end{gathered}
$$

Our first example is a down-and-in barrier call option. Firstly, we consider the case that its barrier $B$ is smaller than its strike price $K$. To receive apositive payoff from the call, the underlying stock price must cross the barrier before maturity and end up above the strike price. We refer to such a situation, that is, $\left\{S_{T}>K \mid \tau_{B} \leq\right.$ $T\}$, as scenario [3]. This scenario renders $g$ cut out for the density functionof the stock return at maturity. Keeping these in mind, we can calculate the price for a down-and-in barrier call option at time $\mathrm{t}$ under Scenario [3] for $\xi \neq 0$ (The calculation for $\xi=0$ goes parallel, which we leave out).

$$
\begin{aligned}
& C_{t}^{B(D I)}(K)_{G E V}=e^{-r(T-t)} \int_{\{\text {profit is nonzero }\}}\left(S_{t} e^{u}-K\right)^{+} d P \\
= & e^{-r(T-t)} \int_{[3]}\left(S_{t} e^{u}-K\right)^{+} d P \\
= & e^{-r(T-t)} \int_{\ln \left(K / S_{t}\right)}^{\infty}\left(S_{t} e^{u}-K\right)^{+} g\left(u ; \sigma_{C B S}\right) d u=C D\left(y ; \sigma_{C B S} ; \varphi=1, \eta=1\right)(B<K)
\end{aligned}
$$

Here $C_{t}^{B(D I)}(K)_{G E V}$ represents the price of a down-and-in call option with strike price $K$ and the barrier $B$ at $t$ for the GEV model.

Next we derive the pricing formula for the option when $B \geq K$. This leads us to 
Jeonggyu Huh, Geonwoo Kim

think of three (not necessarily exclusive) scenarios. We call $\left\{S_{T}>K\right\}$ as Scenario [1], $\left\{S_{T}>B\right\}$ as Scenario [2], and $\left\{S_{T}>B \mid \tau_{B} \leq T\right\}$ as scenario [4], the first two of which have been set with no regard for barrier hit.

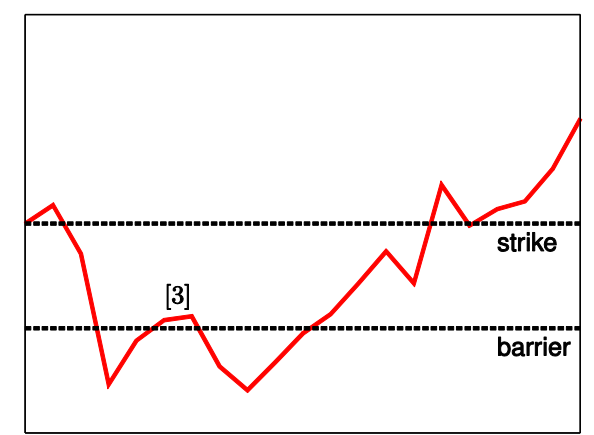

(a) $B<K$

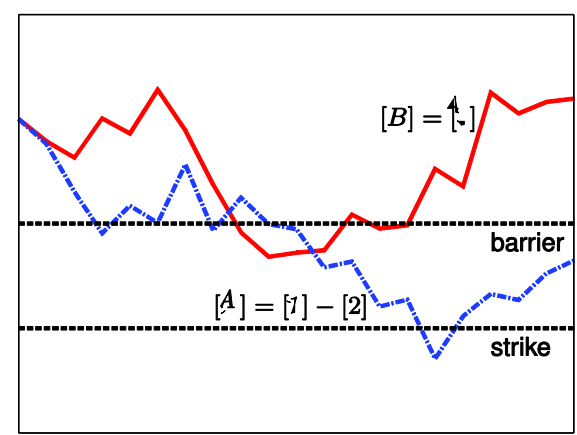

(b) $B \geq K$

Figure 5: scenarios related to a down-and-in call option

Then, the call option generates a payoff exactly when

$$
\begin{gathered}
S_{T}>K \mid \tau_{B} \leq T \Leftrightarrow[\mathrm{A}]\left(K<S_{T} \leq B \mid \tau_{B} \leq T\right) \operatorname{or}[\mathrm{B}]\left(S_{T}>B \mid \tau_{B} \leq T\right) \\
\Leftrightarrow\left(K<S_{T} \leq B\right) \text { or }\left(S_{T}>B \mid \tau_{B} \leq T\right) \\
\Leftrightarrow\left\{[1]\left(S_{T}>K\right) \& \sim[2]\left(S_{T}>B\right)\right\} \text { or }[4]\left(S_{T}>B \mid \tau_{B} \leq T\right)
\end{gathered}
$$

In scenario [4], $g$ plays the role of the density function of the stock return at maturity. However, the scenarios [1] and [2] are extraneous to barrier hit, so it is $f$ that corresponds to the density function of the return at maturity in the scenarios.

$$
\begin{gathered}
C_{t}^{B(D I)}(K)_{G E V}=e^{-r(T-t)} \int_{\{\text {profit is nonzero }\}}\left(S_{t} e^{u}-K\right)^{+} d P \\
=e^{-r(T-t)}\left[\int_{[1]}\left(S_{t} e^{u}-K\right)^{+} d P-\int_{[2]}\left(S_{t} e^{u}-K\right)^{+} d P\right. \\
\left.\quad+\int_{[4]}\left(S_{t} e^{u}-K\right)^{+} d P\right] \\
=e^{-r(T-t)}\left[\int_{\ln \left(K / S_{t}\right)}^{\infty}\left(S_{t} e^{u}-K\right)^{+} f(u) d u-\int_{\ln \left(B / S_{t}\right)}^{\infty}\left(S_{t} e^{u}-K\right)^{+} f(u) d u\right] \\
\quad+\int_{\ln \left(B / S_{t}\right)}^{\infty}\left(S_{t} e^{u}-K\right)^{+} g\left(u ; \sigma_{C B S}\right) d u \\
=C_{t}(K)_{G E V}-\tilde{C}_{t}(K, B)_{G E V}+C D\left(y_{1} ; \sigma ; \varphi=1, \eta=1\right)(B \geq K)
\end{gathered}
$$

Taking advantage of in-out parity, we find the pricing formula of a down-andout barrier call option from those of a vanilla call option and of a down-and-in barrier call option. 
Barrier Option Pricing with Heavy Tailed Distribution

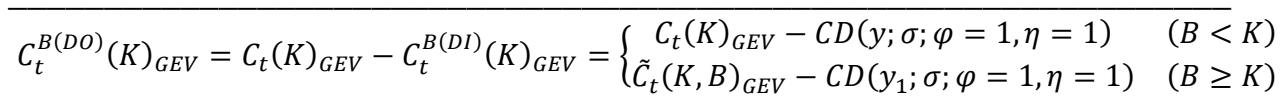

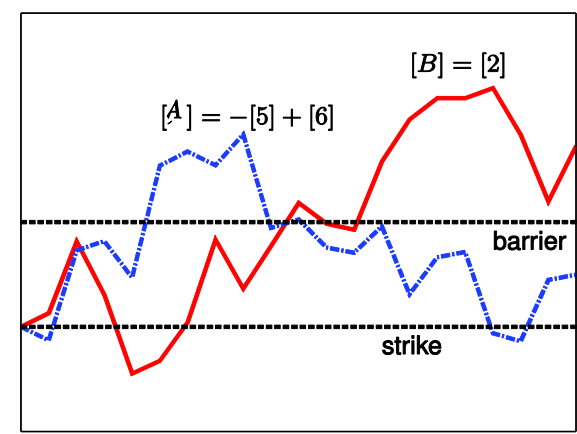

Figure 6: scenarios related to an up-and-in call option

Our next barrier option is an up-and-in call. For $B<K$, the option acts exactly the same as the correspondingvanilla call option does, so all we need to ponder is just estimating the option price for $B \geq K$.As before, the option admits a positive payoff if and only if the underlying price lies above the strike price at maturity while having hit the barrier. So we think about three cases, namely, $\left\{S_{T}>B\right\}$ (Scenario [2]), $\left\{S_{T} \leq K \mid \tau_{B} \leq T\right\}$ (Scenario [5]), and $\left\{S_{T} \leq B \mid \tau_{B} \leq T\right\}$ (Scenario [6]).

$$
\begin{gathered}
S_{T}>K \mid \tau_{B} \leq T \\
\Leftrightarrow[\mathrm{A}]\left(K<S_{T} \leq B \mid \tau_{B} \leq T\right) \text { or }[\mathrm{B}] \Leftrightarrow\left(K<S_{T} \leq B \mid \tau_{B} \leq T\right) \text { or }\left(S_{T}>B\right) \\
\Leftrightarrow\left\{\sim[5]\left(S_{T} \leq K \mid \tau_{B} \leq T\right) \&[6]\left(S_{T} \leq B \mid \tau_{B} \leq T\right)\right\} \text { or }[2]\left(S_{T}>B\right)
\end{gathered}
$$

Being either in Scenario [5] or in Scenario [6] implies that the underlying stock price breaches the barrier before maturity, so $g$ is right for the density function of the stock return at maturity. On the other hand, since Scenario [2] has nothing to do with barrier breach, $f$ is pertinent to the function.

$$
\begin{aligned}
& C_{t}^{B(U I)}(K)_{G E V}=e^{-r(T-t)} \int_{\{\text {profit is nonzero }\}}\left(S_{t} e^{u}-K\right)^{+} d P \\
& =e^{-r(T-t)}\left[-\int_{[5]}\left(S_{t} e^{u}-K\right)^{+} d P+\int_{[6]}\left(S_{t} e^{u}-K\right)^{+} d P+\int_{[2]}\left(S_{t} e^{u}-K\right)^{+} d P\right] \\
& =e^{-r(T-t)}\left[\int_{\ln \left(B / S_{t}\right)}^{\infty}\left(S_{t} e^{u}-K\right)^{+} f(u) d u\right. \\
& \left.-\int_{-\infty}^{\ln \left(K / S_{t}\right)}\left(S_{t} e^{u}-K\right)^{+} g\left(u ; \sigma_{C B S}\right) d u+\int_{-\infty}^{\ln \left(B / S_{t}\right)}\left(S_{t} e^{u}-K\right)^{+} g\left(u ; \sigma_{C B S}\right) d u\right] \\
& =\tilde{C}_{t}(K, B)_{G E V}-C D\left(y ; \sigma_{C B S} ; \phi=1, \eta=-1\right)+C D\left(y_{1} ; \sigma_{C B S} ; \phi=1, \eta=-1\right) \quad(B \geq K)
\end{aligned}
$$

Again, by invoking in-out parity once more, we can obtain the pricing formula of an up-and-out call option from those of a vanilla call option and of an up-and-in call option, which implies that the price of an up-and-out call option should be zero for $B<K$. In a similar manner, we can draw analytic form solutionsfor barrier put 
option prices, which are elaborated on in Appendix B.

\section{CBS Volatility}

$$
\begin{aligned}
C_{t}^{B(U O)}(K)_{G E V} & =C_{t}(K)_{G E V}-C_{t}^{B(U I)}(K)_{G E V} \\
& =C_{t}(K)_{G E V}-\tilde{C}_{t}(K, B)_{G E V}+C D\left(y ; \sigma_{C B S} ; \phi=1, \eta=-1\right) \\
& -C D\left(y_{1} ; \sigma_{C B S} ; \phi=1, \eta=-1\right) \quad(B \geq K)
\end{aligned}
$$

This section aims to show that the CBS model serves as a bridge between the GEV model and the BS model. Thus it is essential that we make a formal definition of a CBS volatility.

As has seen earlier, a vanilla option price is virtually determined by a central neighborhood of the return distribution. This suggests that the price obtained with the GEV model is almost the same as that with the CBS model, owing to Assumption 2. Thus, according to the fact that any vanilla option has a positive vega in the BS model, there is, once the existence is guaranteed, a unique solution $\sigma_{C B S}$ for $C_{t}(K)_{G E V}=C_{t}(K)_{C B S}$ with respect to the variable $\sigma$. It is the very solution that we refer to as the CBS volatility henceforward. We remark that upon determined, $\sigma_{C B S}$ is also the unique solution of $P_{t}(K)_{G E V}=P_{t}(K)_{C B S}$ by virtue of put-callparity. The CBS volatility defined this way depends on the strike price of the option.

In the same vein, we can also devise the CBS volatility when it comes to barrier option pricing. But in general, a naive application of the above volatility may incur the discontinuity of the fair pricing solution in $B$. Hence so as to reflect the continuity of fair prices in a market, we ought to adapt the definition of CBS volatility for practical use. As will be seen later, such alteration takes place by means of the quantities $C_{t}(K), P_{t}(K)$ plus $\tilde{C}_{t}(K, B), \tilde{P}_{t}(K, B)$. Fortunately, the CBS volatility defined for a barrier option also functions as a link between the two models, and the intuitive interpretation of the definition sounds rather plausible because $\tilde{C}_{t}(K, B), \tilde{P}_{t}(K, B)$ are barely different from vanilla option prices provided that $B$ is near $K$. In the rest of this section, we will prove that the pricing functions for barrier options based on the CBS volatility are continuous with respect to $B$ regardless of barrier option type.

(a) Definition of the CBS volatility

The CBS volatility is defined to be the solution of one of the following equations: it depends on the barrier, the strike price, and the type of the barrier option concerned.

\begin{tabular}{|c|c|c|}
\hline $\begin{array}{c}\text { The type of barrier } \\
\text { option }\end{array}$ & $K<S_{t}$ & $K \geq S_{t}$ \\
\hline \hline down type call & $\tilde{C}_{t}\left(K, S_{t}\right)_{G E V}=\tilde{C}_{t}\left(K, S_{t}\right)_{C B S}$ & $C_{t}(K)_{G E V}=C_{t}(K)_{C B S}$ \\
\hline up type call & $C_{t}(K)_{G E V}-\tilde{C}_{t}\left(K, S_{t}\right)_{G E V}=C_{t}(K)_{C B S}-\tilde{C}_{t}\left(K, S_{t}\right)_{C B S}$ \\
\hline down type put & $P_{t}(K)_{G E V}-\tilde{P}_{t}\left(K, S_{t}\right)_{G E V}=P_{t}(K)_{C B S}-\tilde{P}_{t}\left(K, S_{t}\right)_{C B S}$ \\
\hline up type put & $P_{t}(K)_{G E V}=P_{t}(K)_{C B S}$ & $\tilde{P}_{t}\left(K, S_{t}\right)_{G E V}=\tilde{P}_{t}\left(K, S_{t}\right)_{C B S}$ \\
\hline
\end{tabular}

The definitions of $P_{t}(K)_{G E V}, \tilde{P}_{t}(K, B)_{G E V}$ are introduced in Appendices A and B.

(b) Theorem on the CBS volatility

Regardless of the type, the barrier option pricing function is continuous at all 
Barrier Option Pricing with Heavy Tailed Distribution

barrier values.

(Proof) Here we only verify the continuity of the pricing function for a down-andin barrier call option. The ways to prove our statement for the other types are basically similar to this. Because the CBS model is one of BS models, the pricing formula for a down-and-in call option in the CBS model could be deduced from that in the BS model. ${ }^{10}$

$$
C_{t}^{B(D I)}(K)_{C B S}=\left\{\begin{array}{cr}
C D\left(y ; \sigma_{C B S} ; \phi=1, \eta=1\right) & (B<K) \\
C_{t}(K)_{C B S}-\tilde{C}_{t}(K, B)_{C B S}+C D\left(y_{1} ; \sigma_{C B S} ; \phi=1, \eta=1\right) & (B \geq K)
\end{array}\right.
$$

Because of the continuity of $C_{t}^{B(D I)}(K)_{C B S}$, it is enough to show that $C_{t}^{B(D I)}(K)_{G E V}-C_{t}^{B(D I)}(K)_{C B S}$ iscontinuous. Since the continuity of $C_{t}^{B(D I)}(K)_{C B S}$, except at $B=K$ and $B=S_{t}$ is already known, we needto check whether the difference is continuous at both the points.

Calculating $C_{t}^{B(D I)}(K)_{G E V}-C_{t}^{B(D I)}(K)_{C B S}$,

$C_{t}^{B(D I)}(K)_{G E V}-C_{t}^{B(D I)}(K)_{C B S}=\left\{\begin{array}{cc}0 & (B<K) \\ {\left[C_{t}(K)_{G E V}-C_{t}(K)_{C B S}\right]-\left[\tilde{C}_{t}(K, B)_{G E V}-\tilde{C}_{t}(K, B)_{C B S}\right]} & (B \geq K)\end{array}\right.$

When $B=K, C_{t}(K)=\tilde{C}_{t}(K, B)$, so

$$
\lim _{B \rightarrow K^{-}}\left[C_{t}^{B(D I)}(K)_{G E V}-C_{t}^{B(D I)}(K)_{C B S}\right]=0=\left[C_{t}^{B(D I)}(K)_{G E V}-C_{t}^{B(D I)}(K)_{C B S}\right]_{B=K}
$$

Therefore $C_{t}^{B(D I)}(K)_{G E V}$ is continuous at $B=K$. Now let us show the continuity of $C_{t}^{B(D I)}(K)_{G E V}$ at $B=S_{t}$ is continuous. Note that $B=S_{t} \Rightarrow C_{t}^{B(D I)}(K)=$ $C_{t}(K)$, so if $K<S_{t}$.

$\lim _{B \rightarrow S_{t^{-}}}\left[C_{t}^{B(D I)}(K)_{G E V}-C_{t}^{B(D I)}(K)_{C B S}\right]=\left[C_{t}(K)_{G E V}-C_{t}(K)_{C B S}\right]-\left[\tilde{C}_{t}(K, B)_{G E V}-\tilde{C}_{t}(K, B)_{C B S}\right]$

$$
=C_{t}(K)_{G E V}-C_{t}(K)_{C B S}=\left[C_{t}^{B(D I)}(K)_{G E V}-C_{t}^{B(D I)}(K)_{C B S}\right]_{B=S_{t}}
$$

where the penultimate equality follows from our definition of CBS volatility for $K<S_{t}$. This proves our assertion for $K<S_{t}$. When $K \geq S_{t}$,

$$
\begin{aligned}
\lim _{B \rightarrow S_{t^{-}}} & {\left[C_{t}^{B(D I)}(K)_{G E V}-C_{t}^{B(D I)}(K)_{C B S}\right]=0 } \\
= & C_{t}(K)_{G E V}-C_{t}(K)_{C B S}=\left[C_{t}^{B(D I)}(K)_{G E V}-C_{t}^{B(D I)}(K)_{C B S}\right]_{B=S_{t}}
\end{aligned}
$$

where the last equality but one also holds due to the definition. These conclude our proof.

\section{Numerical Experiment}

In general, the trading volume of OTC derivatives is relatively low compared to that of ET derivatives. What is more, little data on any trade of OTC derivatives can be found because such trades are arranged "over the counter." Thus, in this chapter, we justify our previous assertions via numerical experiments based on the

\footnotetext{
${ }^{10}$ If we denote by $f_{B S}(u ; \sigma)$ the density function of the BS model, then $f_{C B S}(u)=f_{B S}\left(u ; \sigma_{C B S}\right)$ And $C_{t}(K)_{C B S}=\int_{\ln \left(K / S_{t}\right)}^{\infty}\left(S_{t} e^{u}-K\right)^{+} f_{C B S}(u) d u, \tilde{C}_{t}(K, B)_{C B S}=\int_{\ln \left(B / S_{t}\right)}^{\infty}\left(S_{t} e^{u}-K\right)^{+} f_{C B S}(u) d u$.
} 
Heston mode ${ }^{11}$. A brief direction for the experiment goes as follows:

1. Make a random determination on the parameters $\kappa, \theta, \sigma, \rho, v_{0}$ of the Heston model, the risk-neutral interest rate $r$, and the time to maturity $\tau$.

2. Under the parameters determined in 1, produce the prices of vanilla options over strike price. ${ }^{12}$

3. Under the same parameters, perform barrier option pricing over strike price and barrier. ${ }^{13}$

4. Estimate the parameters of both the BS model and the GEV model from the data in 2.

5. Price the barrier options in both ways with the parameters from 4 . Then compare these data sets with those in 3.

6. Repeat the above steps with replacing the parameters in 1 by other values.

Since the Heston model is flexible due to the abundance of parameters, it is believed that it can reproduce a variety of risk-neutral measures (or, densities). Taking this feature and the scarcity of pertinent real market data into consideration, we generated data sets from the Heston model as a substitute for the corresponding real market data. One might call this setting into question: why not using the Heston model for evaluation in the first place? However, it is a different matter from that to utilize the data after having the parameters suited for the real market. To be more precise, the Heston model fitting with no error will of course price barrier options much more correctly than the GEV model. In reality, however, as parameter estimation always entails errors, high flexibility of a model does not necessarily guarantee its accuracy: recall that the more parameters get involved, the more subject the model is to error.

Figure 7 depicts implied volatility curves derived from both models in a (virtual) market with current stock price at 100 . Here, the stability of a market is classified in inverse proportion to the curvature of its implied volatility curve. As can be seen, in an unstable market, the numerical results of the GEV model fit better with the curve given by the Heston model than those of the BS model. On the other hand, in a stable market, the latter approximation do better: this can be inferred from that the GEV model provides anything but horizontal implied volatility curves, while those from the BS model must be constant.

\footnotetext{
${ }^{11}$ Heston(1993) suggested a model where $S_{t}$ satisfies the followings:

$$
\frac{d S_{t}}{S_{t}}=r d t+\sqrt{V_{t}} d W_{t}{ }^{S}, d V_{t}=\kappa\left(\theta-V_{t}\right) d t+\sigma \sqrt{V_{t}} d W_{t}{ }^{V}, d W_{t}^{S} d W_{t}^{V}=\rho d t
$$

Using the correlation coefficient $\rho$, this model reflects leverage effect between the stock price and the volatility.

${ }^{12}$ Heston(1993) demonstrated a way to price a vanilla option via an analytic formula with a complex integral. Later Albercher(2006) found another way in order to get around a numerical issue emerging from the integral.

${ }^{13}$ The options were priced via an application of finite difference method to the PDE induced from the Heston model made in 1.
} 
Throughout the experiments, we endeavored to adjust the strike prices to riskneutral return distributions. For example, even with the same strike price, how deep out of the money a put option is can alter in line with the return distribution, which supports the plausibility of such adjustments. To realize this, we took the following steps.

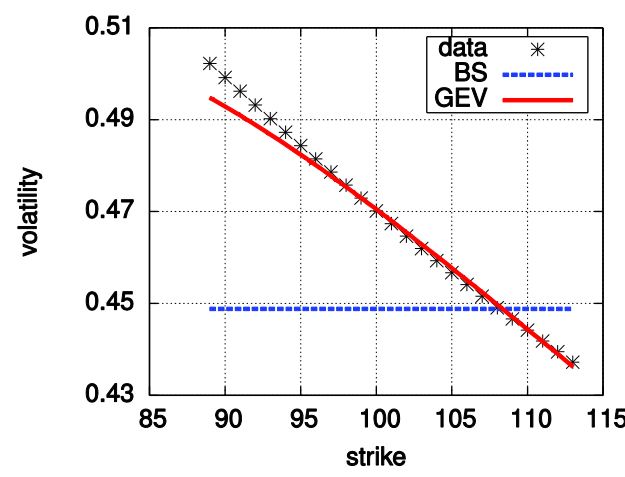

(a) unstable market

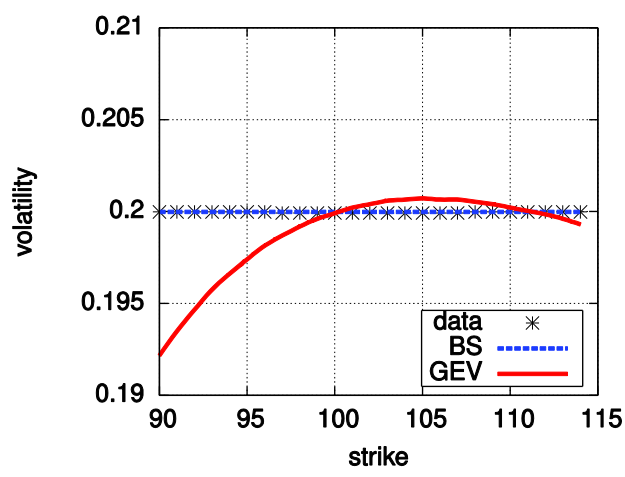

(b) stable market

Figure 7: BS-based and GEV-based implied volatility curves

Firstly, we ran Monte Carlo simulations in order to approximate $P\left(S_{T} \leq x\right)$, the cumulative density function of $S_{T}$. Next we defined $P_{d}(K)$ and $P_{u}(K)$ by

$$
P_{d}(K)=\frac{P\left(S_{T} \leq K\right)}{P\left(S_{T} \leq S_{t}\right)}, P_{u}(K)=\frac{P\left(S_{T} \geq K\right)}{P\left(S_{T} \geq S_{t}\right)}
$$

One can take notice that the smaller $P_{d}(K)\left(P_{u}(K)\right.$, respectively) gets, the deeper out of the money (deep in the money, respectively) an option becomes. Finally we took $K$ so as to fix $P_{d}$ or $P_{u}$ for each experiment.

Figure 8 graphs the prices of up-and-in barrier put options over barrier in an unstable market. As can be seen, the GEV model prices the options more closely to the Heston prices than the BS model does. There are some instances wherein the GEV barrier option pricing is nearly no different from the BS pricing, but at least no worse than that. Likewise, this effectiveness takes place for other kinds of barrier options. Figure 9 compares the numerical results of pricing down-and-in barrier options in a stable market. In contrast with the previous case, on the whole, the BS option pricing outperforms the other, and the same is true of other types as well. Still, the discrepancy is less significant than the efficiency observed above ${ }^{14}$.

\footnotetext{
${ }^{14}$ In the unstable market, the estimated average L2 error for the BS method was 2.695, whereas that for the GEV method was 1.369, almost half of it. In the stable market, however, the error for the BS
} 
In other words, it can be said that the GEV evaluation comes close to the BS evaluation.

This section has recorded the results from only two choices for parameter sets which represent the markets in different situations. But this conclusion was made on the basis of experiments with more than a hundred of different settings; they produced similar results. Obviously, such inductive inference cannot assure the conclusion, but the results gained thus far apparently proposes that barrier options are more fairly priced by the GEV model than by the counterpart when the market involved is unstable.
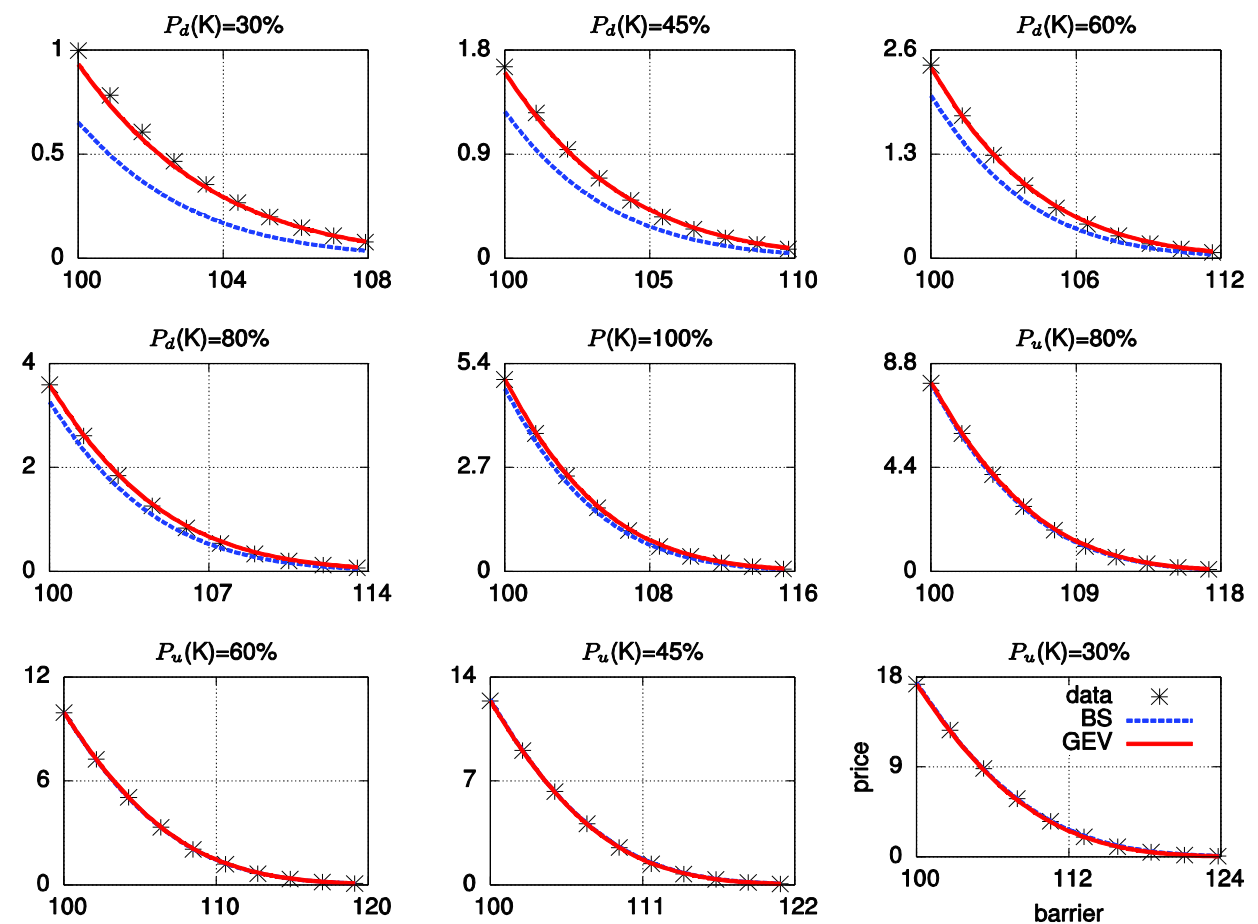

Figure 8: BS pricing vs. GEV pricing in the unstable market (up-and-in put option)

\section{Concluding Remarks}

Markose and Alerton (2011) derived no-arbitrage conditions and pricing equations of vanilla options under the assumption that the simple negative returns of underlying asset follow GEV distribution. They left some equations unsolved and made some minor mistakes. In the appendix, we derive missing equations and rectify errors. We use Rubinstein and Reiner's technique and the GEV model to

method was 0.056 , while that for the GEV method was 0.545 . Despite this conspicuous ratio between them, the error sizes are so small that both model can be considered to have approximated the prices well. It thus can be inferred from this that the GEV option pricing still does fine in a stable market. 
Barrier Option Pricing with Heavy Tailed Distribution

derive closed-form solutions of barrier options.

During the derivation, we suppose that the central neighborhood of the GEV distribution and the normal distribution are similar by introducing the Corrected BS (CBS) model - the BS model close to the GEV model - to make our assumption
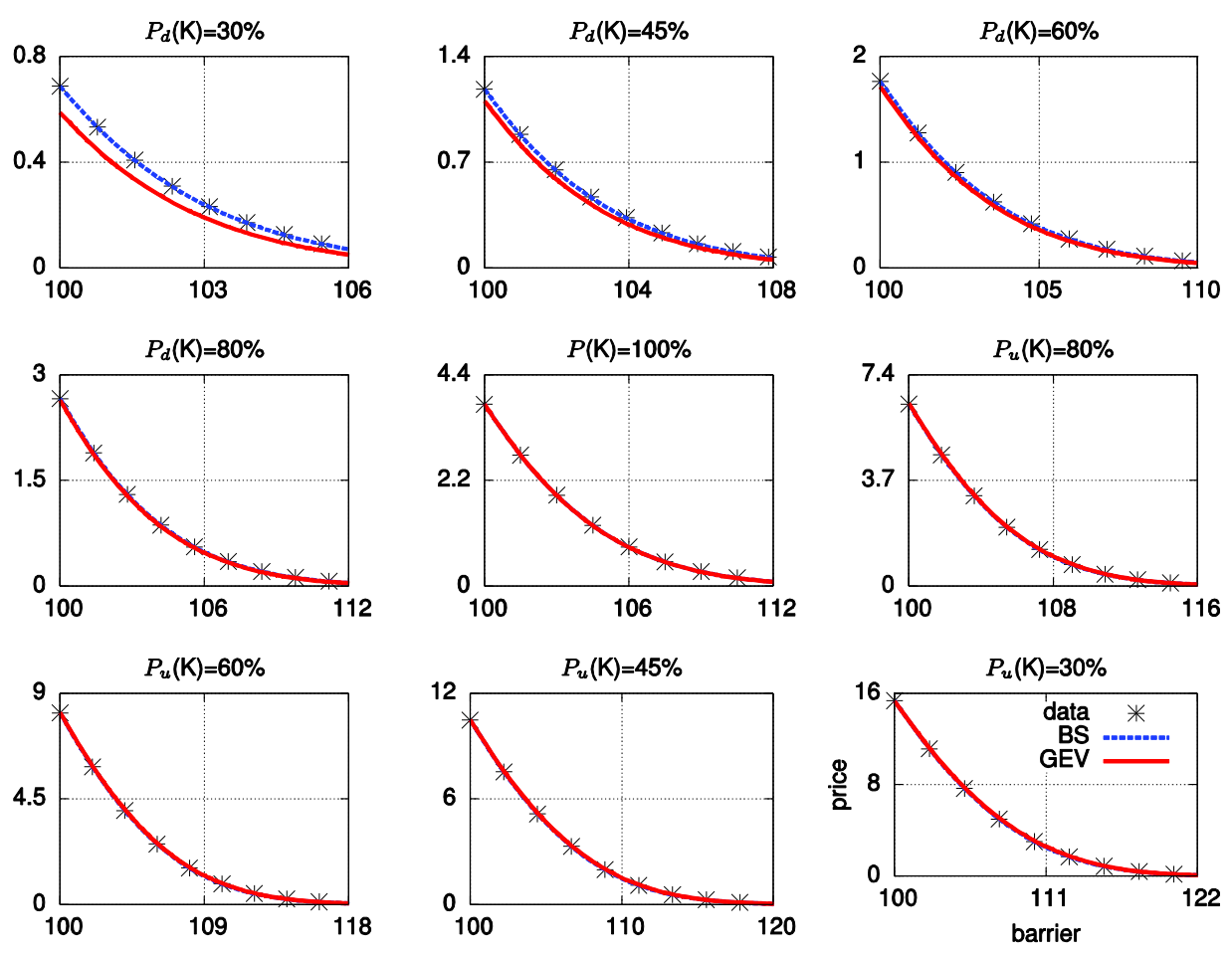

Figure 9: BS pricing versus GEV pricing in the stable market (up-and-in put option)

reasonable. The CBS volatility can be chosen arbitrarily depending on the situation but we fixed it to make barrier option prices continuous to barriers under GEV model. It uniquely exists on high probability. Besides, the numerical experiments based on Heston model tells that having one parameter more, the new model surpassed the BS model when it comes to barrier option pricing.

People still use the BS model believing that the BS model reflects the market, even though the BS model does not reflect the market perfectly. However, when the market is unstable, the BS model has low fittingability due to the skewness and the kurtosis of the return distribution. In this paper, we adjust the skewness and the kurtosis of the distribution of stocks in the global credit crisis by only adding one parameter with the GEV model. We prove that the BS model undervalues vanilla call options and more undervalues in-type barrier options. We also find that the probability of large pricing gap of OTC derivatives between two probability 
Jeonggyu Huh, Geonwoo Kim

distributions increases, the one derived from the BS model and the other from the GEV model, even if the evaluation of exchange-traded derivatives are similar. These analyses may imply that the BS model overvalues or undervalues prices of OTC derivatives regardless of a market situation and can even bring wrong prices of derivatives when the market is unstable.

\section{ACKNOWLEDGEMENTS}

This work was supported by the National Research Foundation of Korea grant funded by the Korea government (Grant No. NRF-2017R1E1A1A03070886).

\section{REFERENCES}

[1] Black, F., Scholes, M. (1973), The Pricing of Options and Corporate Liabilities. The Journal of Political Economy 81(3), 637-654;

[2] Embrechts, P., Klüppelberg, C., Mikosch, T. (1997), Modelling Extremal Events for Insurance and Finance. Springer, New York;

[3] Gabaix, ,X., Gopikrishnan, P., Plerou, V., Stanley H. (2003), A Theory of Power-Law Distributions in Financial Market Fluctuations. Nature 423, 267-270; [4] Harrison, J., Pliska, S. (1981), Martingales and Stochastic Integrals in the Theory of Continuous Trading. Stochastic Processes Application 11, 215-260; [5] Hosking, J., Wallis, J. (2005), Regional Frequency Analysis: An Approach Based on L-moments. Cambridge, Cambridge University Press;

[6] Jackwerth, J. C. (1999), Option Implied Risk-Neutral Distributions and Implied Binomial Trees: A Literature Review. The Journal of Derivatives 7, 66-82; [7] Jackwerth, J. C., Rubinstein, M. (1996), Recovering Probability Distributions from Option Prices. The Journal of Finance, 51(5), 1611-1631; [8] Kim, J., Kim, H.-O. (2014), Option Implied Tail Index and Volatility Based on Heavy-tailed Distributions: Evidence from KOSPI 200 Index Options Market. Global Economic Review, 24(3), 269-284;

[9] Mandelbrot, B. (1963), The Variation of Certain Speculative Prices. Journal of Business 36, 394-419;

[10] Markose, S., Alentorn, A. (2011), The Generalized Extreme Value

Distribution, Implied Tail Index, and Option Pricing. Journal of Derivatives 18(3), 35-60;

[11] Merton, R.C. (1973), Theory of Rational Option Pricing. The Bell Journal of Economics and Management Sciences 4(1), 141-183;

[12] Rubinstein, M., Reiner, E. (1991), Breaking Down the Barriers. Risk 4(8), 28-35;

[13] Shreve, S. (2008), Stochastic Calculus for Finance II: Continuous Time

Models. New York, Springer;

[14] Heston, S. L. (1993), A Closed-Form Solution for Options with Stochastic Volatility with Applications to Bond and Currency Options. Review of financial studies, 6(2), 327-343;

[15] Albrecher, H., Mayer, P., Schoutens, W. \&Tistaert, J. (2007), The Little 
Barrier Option Pricing with Heavy Tailed Distribution

Heston Trap. Willmott Magazine, 83-92.

\section{Appendix A - The price formula of vanilla put options for the GEV model ${ }^{15}$}

Because we already know the price formula of vanilla call options for the GEV model, by using put-call parity, we can easily derive the price formula of vanilla put options as follows.

$$
P_{t}(K)_{G E V}=C_{t}(K)_{G E V}-S_{t}+e^{-r(T-t)} K
$$

Here $P_{t}(K)_{G E V}$ is the price of a vanilla put option with the strike price $K$ at $t$ for the GEV model.

(i) The price formula of vanilla put options for the GEV model where $\xi \neq 0$

$$
\begin{aligned}
P_{t}(K)_{G E V}=e^{-r(T-t)}\left[K\left(1-e^{-H^{-1 / \xi}}\right)\right. & \\
& \left.-S_{t}\left(e^{r(T-t)}-\left(1-\mu+\frac{\sigma}{\xi}\right) e^{-H^{-1 / \xi}}+\frac{\sigma}{\xi} \Gamma\left(1-\xi, H^{-1 / \xi}\right)\right)\right] \\
H=1+ & \frac{\xi}{\sigma}\left(1-\frac{K}{S_{t}}-\mu\right), \mu=1-\frac{F_{t}^{T}}{S_{t}}-\left(\frac{\Gamma(1-\xi)-1}{\xi}\right) \sigma
\end{aligned}
$$

(ii) The price formula of vanilla put options for the GEV model where $\xi=0$

$$
\begin{aligned}
P_{t}(K)_{G E V} & =e^{-r(T-t)}\left[K\left(1-e^{-\exp \bar{H}}\right)-S_{t}\left(e^{r(T-t)}-(1-\mu+\bar{H} \sigma) e^{-\exp \bar{H}}+\sigma \Gamma(0, \exp \bar{H})\right)\right] \\
\bar{H} & =-\frac{1-K / S_{t}-\mu}{\sigma}, \mu=1-\frac{F_{t}^{T}}{S_{t}}-\tilde{\gamma} \sigma \quad(\tilde{\gamma}: \text { the Euler-Mascheroni constant })
\end{aligned}
$$

Appendix B - The price formula of barrier put options for the GEV model where $\xi \neq \mathbf{0}$.

Applying the similar method used to derive the price formula of vanilla put options for the GEV model, the below integrals could be explicitly calculated. Let the result be defined as $\tilde{P}(K, B)_{G E V}$.

* An ancillary term for the analytic form valuation of barrier put options for the GEV model

$$
\begin{aligned}
\widetilde{P}_{t}(K, B)_{G E V} & =e^{-r(T-t)} \int_{-\infty}^{\ln \left(B / S_{t}\right)}\left(K-S_{t} e^{u}\right)^{+} f(u) d u \\
& =e^{-r(T-t)}\left[K\left(1-e^{-H_{1}^{-1 / \xi}}\right)-S_{t}\left(e^{r(T-t)}-\left(1-\mu+\frac{\sigma}{\xi}\right) e^{-H_{1}^{-1 / \xi}}+\frac{\sigma}{\xi} \Gamma\left(1-\xi, H_{1}^{-1 / \xi}\right)\right)\right](\xi \neq 0)
\end{aligned}
$$

Here $H_{1}=1+(\xi / \sigma)\left(1-B / S_{t}-\mu\right)$.Note that $P_{t}(K)=\tilde{P}_{t}(K, B)$ if $B \geq K$.

And the function $C D$ and the constants $y, y_{1}$ defined in the Section 3 are also used

\footnotetext{
${ }^{15}$ Markose(2011) derives the price formula of vanilla put options for the GEV model only if $\xi>0$.
} 
Jeonggyu Huh, Geonwoo Kim

here.

$$
\begin{gathered}
C D(x ; \sigma, \phi, \eta)=\phi S_{t}\left(B / S_{t}\right)^{2 \lambda} N(\eta x) \\
-\phi K e^{-r(T-t)}\left(B / S_{t}\right)^{2 \lambda-2} N(\eta x-\eta \sigma \sqrt{T-t}) \\
y=\frac{\ln \left(B^{2} / S_{t} K\right)}{\sigma \sqrt{T-t}}+\lambda \sigma \sqrt{T-t}, y_{1}=\frac{\ln \left(B / S_{t}\right)}{\sigma \sqrt{T-t}}+\lambda \sigma \sqrt{T-t}, \lambda=\frac{1}{2}+\frac{r}{\sigma^{2}}
\end{gathered}
$$

Since the detail of the derivation is similar to that of call options, it is omitted here. If one likes to derive the price formula of vanilla put options, he is advised to refer to the Section 3 and Rubinstein(1991) together.

(i) The price formula of down-in put options for the GEV model where $\xi \neq 0$

$$
\begin{aligned}
& P_{t}^{B(D I)}(K)_{G E V} \\
& =\left\{\begin{array}{rr}
\tilde{P}_{t}(K, B)_{G E V}+C D\left(y ; \sigma_{C B S} ; \phi=-1, \eta=1\right)-C D\left(y_{1} ; \sigma_{C B S} ; \phi=-1, \eta=1\right) & (B<K) \\
P_{t}(K)_{G E V} & (B \geq K)
\end{array}\right.
\end{aligned}
$$

(ii) The price formula of down-out put options for the GEV model where $\xi \neq 0$

$P_{t}^{B(D O)}(K)_{G E V}$

$=\left\{\begin{array}{r}P_{t}(K)_{G E V}-\tilde{P}_{t}(K, B)_{G E V}-C D\left(y ; \sigma_{C B S} ; \phi=-1, \eta=1\right)+C D\left(y_{1} ; \sigma_{C B S} ; \phi=-1, \eta=1\right) \\ (B<K) \\ (B \geq K)\end{array}\right.$

(iii) The price formula of up-in put options for the GEV model where $\xi \neq 0$

$$
P_{t}^{B(U I)}(K)_{G E V}=\left\{\begin{array}{rr}
P_{t}(K)_{G E V}-P_{t}(B)_{G E V}-C D\left(y_{1} ; \sigma_{C B S} ; \phi=-1, \eta=-1\right) & (B<K) \\
C D\left(y ; \sigma_{C B S} ; \phi=-1, \eta=-1\right) & (B \geq K)
\end{array}\right.
$$

(iv) The price formula of up-out put options for the GEV model where $\xi \neq 0$

$$
P_{t}^{B(U O)}(K)_{G E V}=\left\{\begin{array}{l}
P_{t}(B)_{G E V}+C D\left(y_{1} ; \sigma_{C B S} ; \phi=-1, \eta=-1\right)(B<K) \\
P_{t}(K)_{G E V}-C D\left(y ; \sigma_{C B S} ; \phi=-1, \eta=-1\right)(B \geq K)
\end{array}\right.
$$

